Research report

\title{
The scent of the other women: Body odor-induced behavioral and physiological effects on face categorization
}

\author{
Smiljana Mutic ${ }^{\mathrm{a}, \mathrm{b}, 1, *}$, Jessica Freiherr ${ }^{\mathrm{c}, \mathrm{d}, 1}$, Annachiara Cavazzana ${ }^{\mathrm{e}, \mathrm{f}}$, Marta Rocha ${ }^{\mathrm{g}, \mathrm{h}}$, \\ Sandra C. Soares ${ }^{g, h}$, Valentina Parma ${ }^{\mathrm{b}, \mathrm{h}, \mathrm{i}, *}$ \\ ${ }^{a}$ Diagnostic and Interventional Neuroradiology, RWTH Aachen University, Aachen, Germany \\ ${ }^{\mathrm{b}}$ Monell Chemical Senses Center, Philadelphia, PA, USA \\ ${ }^{\mathrm{c}}$ Section Neuroscience of Sensory Perception, Department of Psychiatry and Psychotherapy, FAU Erlangen-Nürnberg, Erlangen, Germany \\ ${ }^{\mathrm{d}}$ Department Sensory Analytics, Fraunhofer Institute for Process Engineering and Packaging IVV, Freising, Germany \\ ${ }^{\mathrm{e}}$ Smell \& Taste Clinic, Department of Otorhinolaryngology, TU Dresden, Dresden, Germany \\ ${ }^{\mathrm{f}}$ Gösta Ekman Laboratory, Department of Psychology, Stockholm University, Stockholm, Sweden \\ ${ }^{\mathrm{g}}$ CINTESIS.UA, Department of Education \& Psychology, University of Aveiro, Aveiro, Portugal \\ ${ }^{\mathrm{h}}$ William James Center for Research, Instituto Superior de Psicologia Aplicada, Lisbon, Portugal \\ ${ }^{\mathrm{i}}$ Department of Clinical Neuroscience, Karolinska Institutet, Stockholm, Sweden
}

\section{A R T I C L E I N F O}

\section{Keywords:}

Chemosensation

Intrasexual competition

Olfaction

Chemosignal

Smell

Heart rate

\begin{abstract}
A B S T R A C T
In body odor research, the interaction of female donors and receivers is scarcely investigated. With the aim to investigate effects of female body odor in a competitive context, we tested 51 women divided into two groups (i.e., a competitive and a non-competitive group, based on verbal instructions). Between groups, we explored whether female body odor exposure (vs. masker odor) modulates emotion categorization (via RT variance and distribution) and physiological reactions (via instantaneous heart rate) in a task with dynamic male and female faces as either angry or happy. Women in the competitive group reported to feel more competitive and performed more accurately. They gathered more emotional information to categorize dynamic faces and when additionally exposed to female body odor, they showed a resistance to cardiac deceleration. Lapses of attention (via RT distribution) occurred irrespective of body odor exposure. Our results support the idea that female body odors, presented in a competitive context, contrast cardiac deceleration and, by tendency, modulate emotion categorization. Data are discussed in the context of chemosignaling and social interactions among women.
\end{abstract}

\section{Introduction}

Animal research has shown that olfactory information mediates behavior related to competition and conflict. Female individuals across species avoid direct conflict (i.e., physical aggression), except when protection of their offspring is at stake. During lactation only, female mice engage in territorial aggression to intruding conspecifics [69] - a behavior strictly mediated by chemosignals [6]. Whether the contextual experience of competition is mediated by body odors in humans is to date unclear, especially when women are in focus of the research. Women are better at picking up subtle cues in non-verbal communication (e.g., facial expressions, [26]) and this is also true for chemosensory communication [66]. With chemosensory cues being subtle by nature and with women being better at picking up subtle cues, the question arises whether women show modulated face recognition in the context of competition and chemosignals, both not palpable or visible to the naked eye.

In contrast to male-male interactions, female-female dyads are dense of nonverbal cues [22,58]. Many studies have explored this communicational aspect: Women recognize ambiguous facial expressions more accurately than men (i.e., $50 \%$ of a facial emotion, [26]; among other aspects [8]), women prefer appeasing strategies to maintain a superficial interpersonal harmony during conflicts (e.g., tendand-befriend, [68]), they tend to subtly derogate same-sex competitors and their social status during sexual selection ([7,12,20,57,70,71]), and they scrutinize same-sex individuals more thoroughly (own-sex bias, e.g., in girls [60]), also in relation to slight hormonal changes (e.g., women judged faces of ovulating women as more attractive than those of non-ovulating women, [61]). Their superiority in decoding emotions [75] and subtle social signals has been explained by an overarching

\footnotetext{
* Corresponding authors at: William James Center for Research- Instituto Superior de Psicologia Aplicada, Rua Jardim do Tabaco, 34, Lisbon, Portugal.

E-mail addresses: smutic@dr.com (S. Mutic), vparma@ispa.pt (V. Parma).

${ }^{1}$ Contributed equally.
} 
evolutionary postulate: The primary caretaker hypothesis [4] assumes an innate ability in females to rapidly read emotions of kin in order to facilitate satisfactory care of offspring.

Since women's sensitivity to subtle cues in non-verbal communication is enhanced, it appears plausible to assume that women capitalize on the body odor information of other women. A limited number of studies has suggested that body odors are subtle cues that can have relevance in competition. The modulation of social interactions and/or competitive scenarios through body odors is mostly explored in intersexual body odor communication research. A competitor can be perceived as a threat and body odors are commonly used to signal such olfactory threat.

In the studies reported next, exposure to body odors - from various forms of threat - were used to observe behavioral and central/autonomic nervous effects on judgment of socio-emotional material: Body odors of unknown individuals activated amygdala and insula, areas involved in the so-called fear network [36]. They also activated comparable autonomic responses to aversively conditioned odors (i.e., skin conductance, [13]). More directly, body odors of aggressive men led to prolonged reactions to anxiety-related material [47] and activated the thalamus (indicating a cross-check with other sensory modalities) and the cingulum (known as a part of the neural alarm system; [48]). Body odors of competitive athletes induced higher skin conductance (i.e., arousal) in socially anxious participants [1]. Recently, we revealed effects on face categorization: body odors of fearful individuals affected dynamic facial expression categorization (i.e., morphing from neutral to either angry or happy [63]).

The above mentioned intersexual research on threatening/competitive body odor was a fruitful method of showing that such subtle cues can be used in a competitive context, but it is mostly characterized by male body odor exposure for both sexes. These results may not directly be translated to women smelling other women's body odors.

From an intrasexual perspective, a limited number of studies considered women's body odors in competitive contexts based on the aforesaid female fine tuning to subtle communication and their preference to signal competition implicitly. Woodward, Thompson \& Gangestad [76] showed that women exposed to female body odors implement more assertive responses to other women. This was only investigated in a hypothetical competition scenario via questionnaires. Second, women fixate same-sex competitors longer than available male targets, when exposed to body odor compounds (i.e., androgen-steroid derivative androstadienone; [54]). The two studies suggest that body odors are indeed associated with indirect competitive strategies and altered face processing in competing women. The limited amount of research on female-female chemosensory interaction is exacerbated by many sex-specific methodological aspects. Changes related to hormonal contraception (e.g., see $[16,24,30]$ ), pregnancy, lactation, menopause onset or menstrual cycle phases $[30,64]$ introduce a wealth of confounding factors influencing donation quality. In contrast, women are appealing as receivers as they are reported to be particularly sensitive to common odors (e.g., $[10,17])$ and body odor features (e.g., [56,59]), including emotions (e.g., [15]).

The goal of this experiment was to determine whether women in reproductive age would categorize dynamic facial expressions differently based on a competitive vs. non-competitive mindset and exposure to female body odors. A dynamic emotion categorization task under exposure to body odors of women (masked with a neutral odor) or masker odor alone (based on [63]) was implemented. We performed analyses of response accuracy, response time (RT) and RT dispersion (to assess lapses of attention) associated with odor exposure and a competitive mindset. To determine the attention-related autonomic nervous activity linked to competition and body odor exposure, we measured instantaneous heart rate (IHR). IHR is an autonomic nervous system measure that is known to reveal variations in cognitive effort, such as the allocation of attention following the presentation of affective stimuli [51,52]. Stimuli capturing attention contribute to cardiac deceleration whereas cardiac acceleration (or resistance to deceleration) is linked to defense responses. Despite the exploratory nature of this research, we used the following hypotheses to guide our analyses:

As a manipulation check, we hypothesized a stronger competitive mindset (measured via competitiveness self-rating and response accuracy in the dynamic emotion categorization task) in the competitive vs. non-competitive group.

Then, we expected the exposure to female body odors vs. masker to modulate the speed of emotion categorization in the competitive (but not in the non-competitive) group. Specifically, longer categorization RT - which reflect a greater level of disambiguation in the categorization of the facial expression - were predicted for the competitive group when exposed to the body odor (in line with facial categorization observed under exposure to threatening body odor, [63]).

Additionally, we hypothesized that the competitive groups, ideally under female body odor exposure, would commit more lapses of attention in categorizing emotional faces (via RT distribution). This would be in line with the idea that the presence of a body odor alone can constitute a threatening cue $[13,54]$ and that threatening body odors (i.e., from stressed donors) affect task performance in ways compatible with reduced attentional resources [65].

Lastly, to reveal increased responsiveness to nonverbal cues of potential competitors, we expect a lack of or resistance to cardiac deceleration in competitive women under exposure to female body odor.

\section{Materials and methods}

\subsection{Body odor donation and chemosensory samples}

Body odor from three healthy heterosexual females (age: $M=28.33$ years, $S D=2.31$ years) was collected after a washout phase of seven days, as previously successfully applied previously $([36,38,44])$. During this washout phase, and the consecutive donation phase, no scented personal care products (e.g., perfumes, deodorants, detergents) were used, no armpit shaving, and no consumption of odorous and spicy food (e.g., onion, garlic, curry, asparagus) was allowed. Donors experienced regular menstrual cycles for the past six months and tested ovulation daily via urine samples. None of the donors ovulated during the donation period (as measured via Wondfo, luteinizing hormone ovulation test stripes, $25 \mathrm{mIU} / \mathrm{ml}$ sensitivity). By this means, a higher hormonal stability of the odor samples from nonovulatory menstrual cycle phases was ensured (compared to sampling during ovulatory menstrual cycle phases). The donors' competitiveness towards other women was scored via a questionnaire (adapted version of the Female Competition Questionnaire; [9]) and all three donors scored in the mid-range of female competitive behavior $(M=36$, $S D=8.54$ ). Donors did not display clinically relevant alexithymia (TAS-20: $\mathrm{M}=46, \mathrm{SD}=4.36$; Toronto Alexithymia Scale; [3]), anxiety (STICSA Trait: $\mathrm{M}=32, \mathrm{SD}=7.23$; State and Trait Inventory for Cognitive and Somatic Anxiety; [72]) or aggression traits (STAXI Trait: $\mathrm{M}=20.67, \mathrm{SD}=3.51$ State Trait Anger Expression Inventory; [67]). During eight consecutive days and nights (donation phase), the donors wore shirts with cotton pads (Ultra-Thin Nursing Pads, Gerber Inc., ON, Canada) sewn into the armpit area. Every day, a new t-shirt with insewn cotton pads was provided, stored in re-sealable plastic bags. The shirts served to hold the pads in place and protected the pads from exogenous odor contamination. The t-shirts as well as the bed linens and towels used during the collection were washed with odorless detergent right before the sampling session [55]. Before going to bed each night, donors showered using the odorless products to remove exogenous odor residues and slept in bed alone so that no external fragrances interfered with the body odor collected on the pads. The resulting axillary body odor pads were collected daily. To avoid odor contamination, all pads were handled with odorless surgical gloves and assessed regarding odor intensity and exogenous odorants by three normosmic olfactory assessors trained to detect contamination of body 
odor samples. Pads were included only if the body odor was detectably strong and did not contain any perceivable exogenous odors (such as perfume, smoke or spices, $n=1$ pad was possibly contaminated by perfume and therefore excluded). All pads were stored in coded resealable plastic bags. Once deemed usable, body odor pads were immediately cut in four quadrants and kept frozen $\left(-80^{\circ} \mathrm{C}\right)$ until application to prevent additional bacterial decomposition [31].

\subsection{Receivers}

A total of 65 healthy, heterosexual women provided written informed consent prior to the experiment. All aspects of the study were approved by the Institutional Review Board of the University of Pennsylvania and complied with the Declaration of Helsinki. Using an estimated effect size of $f=0.2$, error probability of $\alpha=0.05$ and power (1- $\beta$ ) of 0.95 for the mixed effects F-tests, the a priori power analysis ( $\mathrm{G}^{*}$ Power, Version 3.1 ; [19]) indicated a total sample size of $n=56$.

All receivers reported neither psychiatric, physical, neurological diseases, nor current medication intake. Via the 16-item identification subtest of the Sniffin' Sticks test $[27,29]$, normal olfactory function was ascertained $(M=13.63 ; S D=1.15 ;$ range $=11-15)$. All receivers scored above the cut-off score of 11 out of 16 (non-competitive group: $M=13.28 ; \quad S D=1.08 ;$ and non-competitive group: $M=13.96$; $S D=1.09$ ), revealing similar olfactory identification skills. Adapting strict inclusion criteria, 14 out of 65 receivers were excluded due to clinically relevant scores in the screening questionnaires for alexithymia (TAS-20, score $\geq 61, \quad \mathrm{n}=3$ ) and anxiety (STICSA-T, score $\geq 43, n=8$ ) and $n=3$ receivers were excluded because they scored above the respective cut-offs for both questionnaires. The rationale behind these decisions was to avoid biases by receivers with the facilitated detection of offensive or potentially threatening cues [28,56] as well as receivers with impaired ability in recognizing facial expressions due to their inability to experience emotions [14,53]. Also, receivers were excluded due to hyposmia $(n=2)$, as measured via the identification subtest of the Sniffin' Sticks [29], and due to technical problems $(n=3)$. The final sample consisted of 51 female receivers, randomly divided in two groups - based on the verbal instructions they received (non-competitive group: $n=26$, age $M=26.18$ years, $\mathrm{SD}=4.67$ years; range $=18-36$ years vs. competitive group: $n=25$, age $\mathrm{M}=26.42$ years, $\mathrm{SD}=4.41$ years; range $=20-34$ years). Via independent $t$-test, a comparison of the female competitiveness scores between the non-competitive group $(\mathrm{M}=24.15, \mathrm{SD}=8.79)$ and the competitive group $(\mathrm{M}=23.88, \mathrm{SD}=10.83)$ revealed no trait differences $(p=.921)$. None of the receivers took hormonal contraception during the past six months and all experienced normal menstrual cycle length. No group differences for emotional perception with alexithymia screening (TAS-20), trait anxiety screening (STICSA), or trait anger screening (STAXI) were observed (via $t$-tests, all $p_{s}>.05$ ).

No variations of subjective negative emotions (via pre-post STAXI and STICSA state questionnaires) between competitive groups interfered with the participants' emotion categorization performance (supplementary material A). None of the receivers had participated as a body odor donor within the present study. Prior to testing, receivers refrained from using scented personal care products for $24 \mathrm{~h}$ and did not eat or drink one hour before the beginning of the experiment.

An illustration of the experimental procedure can be found in Fig. 1. For a short summary, the receivers first completed ratings and questionnaires (i.e., competitiveness rating at baseline, face ratings, odor ratings and questionnaires to assess trait competition, anger, alexithymia, and anxiety). Then, following the verbal delivery of instructions to feel competitive or non-competitive, respectively, receivers from the competitive and non-competitive groups rated their perceived competitiveness post instructions. Subsequently, they were hooked up to the olfactometer and the electrocardiography device and started the dynamic emotion categorization task during odor exposure (masked FBO and masker odor). At the end of the experimental procedure, receivers completed a competitiveness rating, anxiety / anger state questionnaires, and odor discrimination and identification.

\subsection{Body odor stimuli}

The masked female body odors (masked FBO or mFBO) were created by combining one quadrant (one fourth of a pad) from each donor and a clean quadrant into supra-donor stimuli (3 odorous quadrants +1 odorless; $[37,41,44])$. The quadrant from each donor was collected at different time points during the collection period, allowing each receiver to be presented with a unique sample, and therefore reducing potential effects associated with individual odor donors. To prevent body odor from interacting with added fragrances (e.g., as addressed in [2] and [32]) and to avoid conscious detection of the female body odor [55], a neutral olfactory mask ( $100 \mu \mathrm{l}$ of oakmoss; Oakmoss Inco $10 \%$, isopropyl myristate, Givaudan Fragrances Corporation) was applied onto the clean quadrant pad. All applications of oakmoss odor were done using single channel manual pipettes with disposable tips set to $100 \mu \mathrm{l}$. Control stimuli were created by combining four empty, clean pad quadrants with the same amount of oakmoss odor $(100 \mu \mathrm{l})$ on one pad quadrant.

Odor ratings for masked $\mathrm{FBO}$, masker and an odorless control (empty pad with clean air) on a 100-pt visual analogue scale (VAS) were obtained for the odor qualities intensity, pleasantness, familiarity, threat, and disgust (see supplementary material A). Taken together, no significant differences were found for any of the competition groups (via ANOVAs): the masker and the masked FBO were not perceptually different on any of the self-reported odor features (all ps > .068). Both odors were perceptually different from the odorless control/clean air (all $p_{s}<.05$ ).

During the dynamic emotional categorization trials, receivers were repeatedly exposed to either the oakmoss odor (henceforth masker) or to the masked FBO (female body odor pads of three different donors with oakmoss odor) in a within-subject design (18 odor stimulations in total) administered via a computer-controlled olfactometer [39]. They were instructed to inhale through their nose once a green crosshair appeared in the center of the computer screen. Teflon tubings directed the flow of odorous or clean air (CA) through a manifold attached to the receiver's chest into her nostrils. Per trial, only one odor was applied birhinally with a flow of $1.5 \mathrm{l} / \mathrm{min}$ per nostril. The odors were presented for the duration of the presentation of the facial expressions until the receiver responded via button press. As the length of the odor presentation was longer than a natural breath cycle, we preferred to limit nasal discomfort by excluding the measurement of sniff responses. The receivers were instructed to breathe naturally and an experimenter visually controlled that this was the case. Ultimately, heart rate data did not reflect abnormal respiratory behavior (through respiratory sinus arrhythmia). Between odor stimulations, a washout phase of $17 \mathrm{~s}$ of clean air was applied to prevent olfactory adaptation.

Receivers performed a 9-trial, three-alternative forced choice discrimination task in which the masked FBO had to be discriminated from two masker odors. No receiver was able to significantly discriminate the masked FBO above chance level (6 out of 9 correct responses). We conclude that our results do not depend on perceptual differences across odor conditions. Please refer to supplementary material A for detailed analyses. Before debriefing, participants performed the 16-item identification subtest of the Sniffin' Sticks test $[27,29]$ to assess their odor identification skills.

\subsection{Visual stimuli}

In the dynamic emotion categorization task, facial expressions of anger and happiness in three male and three female individuals from the Karolinska Directed Emotional Faces inventory [35] were shown. In total, 72 single categorization sequences were presented. In each single categorization morphing sequence, a neutral facial expression (i.e., $0 \%$ 

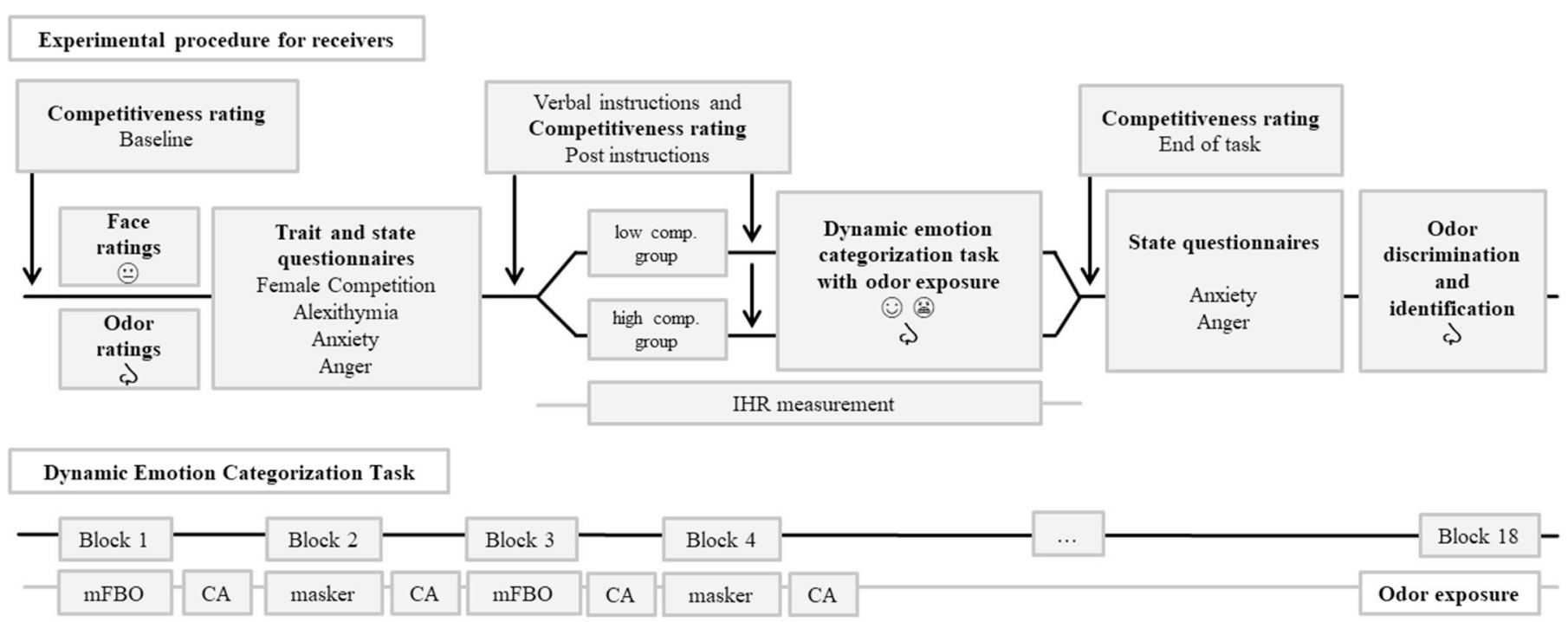

Block à four sequences

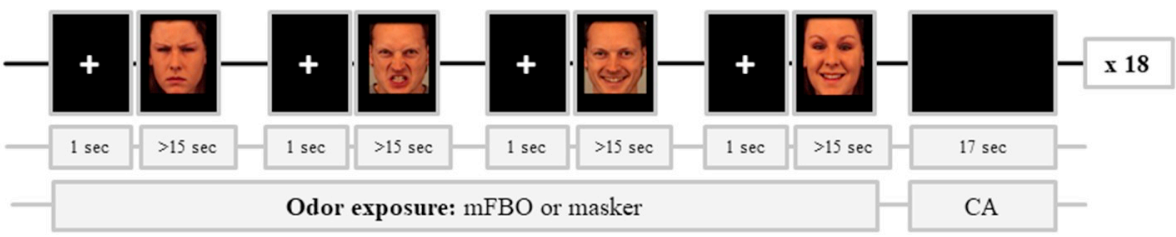

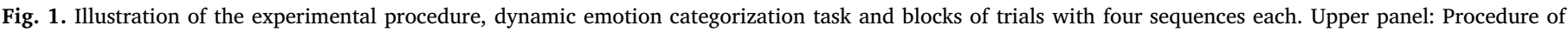

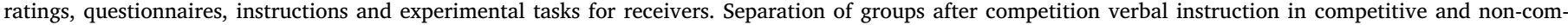

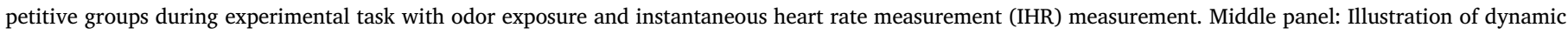

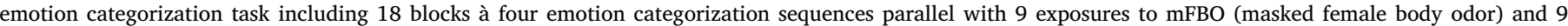

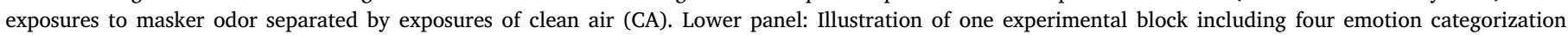

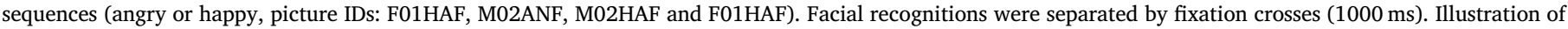
odor exposure and during categorization and olfactory washout of $17 \mathrm{~s}$ of clean air (CA) between blocks presented with a black screen.

angry or happy) gradually changed into a happy (i.e., 100\% happy) or angry (i.e., $100 \%$ angry) facial expression. Thus, the generated intermediate images were changing in 1\%-steps from the neutral facial expression to the full emotional intensity with a total of 100 faces per emotion. The maximum duration of each single morphing sequences was $15 \mathrm{~s}$. The 72 categorization sequences were organized in 18 blocks à four sequences. Only four categorization sequences were put in one block to avoid sensory adaptation or the need for a long experimental session. Within a block, one white fixation cross of $1000 \mathrm{~ms}$ was shown on a black screen. Then, the first of four facial morphing sequences appeared. When receivers gave their response ("angry" or "happy") as fast and as accurate as possible via keyboard press (left and right index fingers on keys "z" or " $m$ "), the facial expressions on the screen disappeared and the second of four morphing sequences appeared on screen. After the fourth morphing face of the block, the odor stimulation ended and $17 \mathrm{~s}$ elapsed on a countdown counter screen. In each block, one olfactory stimulation (masked FBO or masker) was presented constantly while all four emotion categorization sequences were performed. All 18 blocks of four categorizations were separated by a black screen shown for a waiting period of $17 \mathrm{~s}$ (clean air).

In each block, faces gradually morphed into happy faces from a minimum of one to a maximum of three presentations. They were complemented with the necessary number of angry faces to reach four presentations per trial (i.e., one happy face and three angry faces presented in randomized order). Such blocks could have included one to three male or female faces. All combinations of men and women were included to prevent response prediction and to include the whole spectrum of options. For details, please refer to the original morphing procedure [62]. Blocks were presented in a randomized order.

\subsection{Experimental procedure and data analysis}

Receivers were seated in a well-ventilated room with a low amount of external stimulation. An illustration of the experimental procedure can be found in Fig. 1. Initially, they performed a subjective competitiveness rating (baseline) on a 100-pt VAS ranging from not competitive at all to extremely competitive. They then rated male and female faces for visual features (i.e., attractiveness, competitiveness, trustworthiness, threat and dominance) on a 100-pt VAS to rule out facial judgment differences later related to competition (supplementary material A). Receivers further performed perceptual odor ratings (i.e., intensity, pleasantness, familiarity, threat, and disgust) of the masked FBO, the masker and clean air (supplementary material A). The receivers filled out a questionnaire regarding their competitiveness with other women (based on [9]).

Before the experimental trials, receivers completed state questionnaires on anxiety (state version of STICSA) and anger (state version of STAXI, see supplementary material A).

In a between-subject design, receivers were assigned to a high or a low competitiveness group. Two trained experimenters administered the low and high competitiveness instructions. The instructions for the non-competitive group informed the receivers that they needed to identify the facial emotion emerging on the screen as fast and as accurately as possible. The instructions for the competitive group informed the receivers that they needed to respond faster and more accurately than a group of highly competitive individuals who also performed this same task. If they succeeded in being faster and more accurate than the (fictional) group of highly competitive individuals, they would be offered an additional monetary incentive (ultimately, 


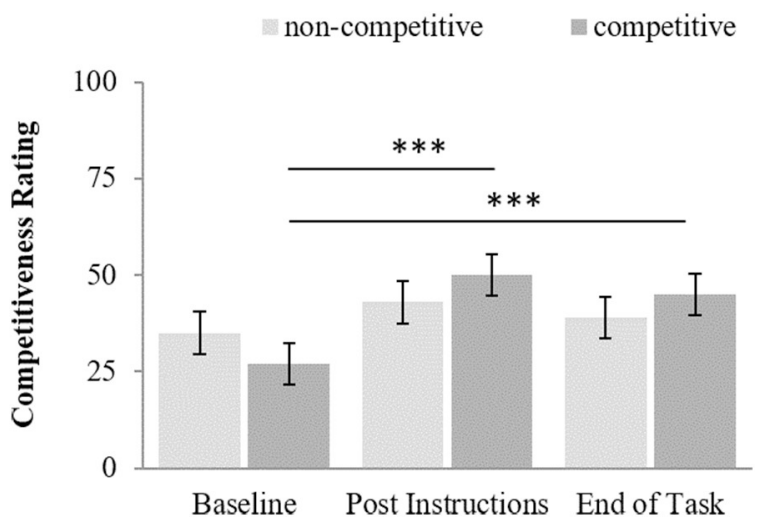

Fig. 2. Competitiveness rating (M and SE) of the non-competitive (light grey) and the competitive (dark grey) groups at baseline, after the competition instructions (post instructions) and at the end of the experimental task. *** marks Bonferroni-corrected significance at $p<.001$.

they received the same amount of money given to the non-competitive participants). Receivers then performed a second subjective competitiveness rating (post-instructions) before starting the experimental task. All receivers were informed that they were going to smell a low intensity natural odor during the experiment. After a practice trial, the receivers performed the dynamic emotion categorization task by indicating the recognized emotion (happy or angry) via button press. The response keys were reversed for half of the receivers in order to prevent lateralization effects. In total, the dynamic emotion categorization task took approximately $20 \mathrm{~min}$. After finishing the task, receivers performed a final subjective competitiveness rating (end of task) and filled out the state questionnaires (anger and anxiety). Upon task completion, receivers were administered a nine-trial, three alternative forced-choice test to assess odor discrimination capacity and performed the olfactory identification test. All receivers were debriefed and were paid 20 dollars after accomplishment of the study. The total duration of the testing was approximately $75 \mathrm{~min}$.

Additional information concerning the heart rate measurement, methods and dependent variables, and data analysis (e.g., outlier labelling) can be found in the supplementary material B. Besides ANOVAs, linear mixed model (LMM) analyses were used where data structure allowed it. To account for distribution skewness, the RT analysis was performed on log-transformed RT data, as per classical guidelines [74]. The analysis of accurate logged RTs was conducted via a restricted maximum likelihood LMM with the fixed effects competition group, odor condition, image category (sex and emotion of the image), and task key inversion. In dynamic emotion categorization tasks, results associated with longer RT represent a greater amount of information gathered to produce a response [63]. To account for the non-independence based on multiple responses by the same subject, we included "subject" as a random effect. Additionally, to test subtler attentional hypotheses on RTs, we evaluated the ex-Gaussian distribution parameters $\mathrm{Mu} \mu$, Sigma $\sigma$, and Tau $\tau$ [5]. RT distribution analyses of central tendency and dispersion via the parameters Mu and Sigma can be found in the supplementary material C. Here, we focus on the parameter Tau describing mean and standard deviation within the exponential part of the function. Tau is indicating whether the pattern of response includes periodic excessive increases in categorization RTs. Tau is argued to be an indicator of poor attention [25].

\section{Results}

3.1. Competitive women felt more competitive and made fewer mistakes in categorizing facial expressions

To check whether competition was successfully induced in the competitive group, a subjective measure (i.e., competitiveness selfrating) and an objective measure (i.e., response accuracy during the categorization task) were analyzed. In the first hypothesis, we expected a higher subjective competitiveness self-rating and a higher response accuracy in the competitive vs. non-competitive group. An ANOVA investigating the dependent variable subjective competitiveness rating on a 100-pt VAS with the between-subject factor competition group and the within-subject factor competitiveness rating at baseline, post instructions, and end of task (with violated sphericity assumptions Mauchly's test: $\chi^{2}(2)=9.050, p=.011$; therefore $\varepsilon=0.853$ ) was performed. A significant main effect of competitiveness rating $\left[\mathrm{F}_{G G}\right.$ $\left.(1,83)=14.918, p<.001, p \eta^{2}=0.233\right]$, no main effect of group $\left[\mathrm{F}_{G G}\right.$ $\left.(1,49)=0.083, p=.775, p \eta^{2}=0.002\right]$, and a significant interaction $\left[\mathrm{F}_{G G}(1,83)=4.122, p=.025, p \eta^{2}=0.078\right]$ was found. Then, further inspection of pairwise comparisons confirmed that the competition groups, as expected, did not differ in competitiveness at baseline [noncompetitive group $\mathrm{M}=34.65, \mathrm{SE}=5.16$ vs. competitive group $\mathrm{M}=27.16, \mathrm{SE}=5.26 ; p=.314]$. Individuals of the non-competitive group did not display rating differences neither baseline vs. post-instructions rating $[\mathrm{M}=42.52, \mathrm{SE}=5.61]$ nor baseline vs. end-of-task rating $[\mathrm{M}=38.62, \mathrm{SE}=5.32]$ nor post-instruction vs. end-of-task rating (all $p_{s}>.086$ ). Compared to baseline, individuals of the competitive group rated their competitive mindset as significantly greater after the instructions $[\mathrm{M}=49.64 ; \mathrm{SE}=5.73]$ and at the end of the experiment $\left[\mathrm{M}=44.96\right.$; $\mathrm{SE}=5.43$, all $p_{s}<.001$; Fig. 2].

Second, the participant's objective compliance with the competition instructions was analyzed by comparing the inaccurate responses per group in the experimental task (i.e., dynamic emotion categorization). The total count of inaccurate responses was higher in the non-competitive group $(6.73 \%$, std. residual $=6.8)$ compared to the competitive group $\left(1.82 \%\right.$, std. residual $\left.=-7.0 ; X^{2}=104.58 ; d f=1 ; p<.001\right)$, indicating that the non-competitive group was less accurate, while the competitive group, specifically instructed to increase performance, was more accurate.

In summary, individuals of the competitive group expressed a stronger subjective competition motivation. By responding more accurately, they were more compliant with the induced competitive framework in contrast to individuals of the non-competitive control group. With regards to both subjective and objective measures, the manipulation was successful.

\subsection{Competitive women invested more time in categorizing faces, also by} tendency for competitive women exposed to female body odor

With regards to the second hypothesis, the specific effects on RT variance in the dynamic emotional categorization task (male and female, angry and happy faces) were analyzed. In the competitive group, exposure to female body odor was expected to be associated with RT differences in contrast to masker odor. The log-transformed RT analysis

\section{Table 1}

Log-transformed RTs, means (and SE), in the dynamic emotion categorization task (values controlled for covariate task key inversion) with all image categories (faces: male angry, male happy, female angry and female happy) by groups (non-competitive vs. competitive) and odor conditions (masked FBO vs. masker). Graphic depiction of Table 1 can be found in supplementary material C. Significant comparisons are flagged via asterisks in Fig. 4.

\begin{tabular}{llllll}
\hline \multirow{2}{*}{ Image category } & \multicolumn{2}{c}{ Masker } & & \multicolumn{2}{c}{ Masked FBO } \\
\cline { 2 - 3 } \cline { 5 - 6 } \cline { 5 - 6 } & Non-competitive & Competitive & & Non-competitive & Competitive \\
\hline Male angry & $7.640(0.088)$ & $7.735(0.081)$ & $7.754(0.089)$ & $7.907(0.080)$ \\
Male happy & $7.429(0.083)$ & $7.354(0.076)$ & & $7.366(0.083)$ & $7.445(0.076)$ \\
Female angry & $7.657(0.087)$ & $7.701(0.077)$ & $7.681(0.087)$ & $7.823(0.076)$ \\
Female happy & $7.464(0.085)$ & $7.571(0.077)$ & $7.440(0.086)$ & $7.632(0.077)$ \\
\hline
\end{tabular}


A

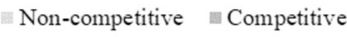

B

$\unrhd$ Masker $\| \mathrm{mFBO}$
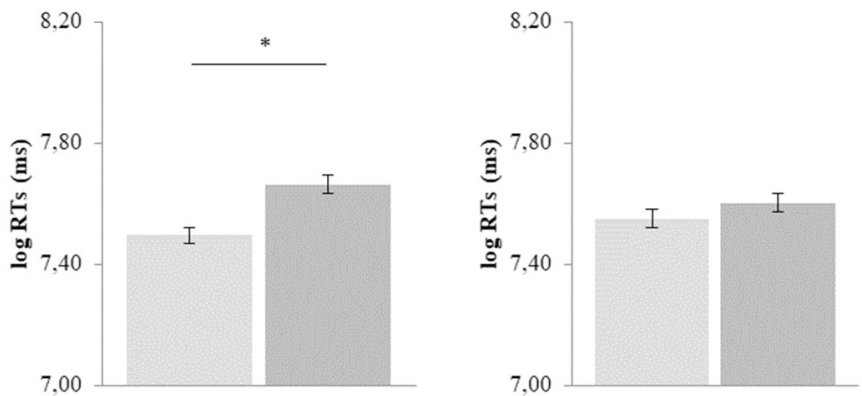

C

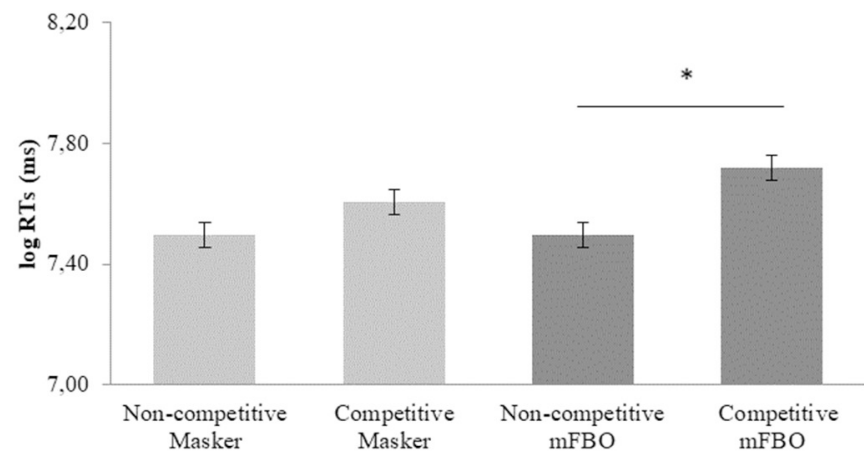

Fig. 3. Log-transformed RTs (M and SE) in the dynamic emotion categorization task. Main effects for competition group (panel A: non-competitive vs. competitive group), odor (panel B: masker vs. masked $\mathrm{FBO}$ [mFBO]) and the explorative result for the two factors combined (panel C). * marks significance at $p<.05$. of accurate responses was conducted via an LMM and yielded main effects of induced competition (non-competitive vs. competitive group, $[\mathrm{F}(1,3319)=55.212, p<.001]$ ), odor exposure (masker vs. masked FBO, $\quad[\mathrm{F}(1, \quad 3319)=6.625, \quad p=.010]), \quad$ and emotion $[\mathrm{F}$ $(1,3319)=5.968, p=.015$; Table 1$]$, but neither for sex of the image $[\mathrm{F}(1,3319)=1.713, p=.191]$ nor statistically significant for the interaction of sex and emotion of the image $[F(1,3319)=3.144$, $p=.076]$. Further effects and interactions outside the focus of the specific research questions are reported in the supplementary material $C$ (all other interactions: $p_{s}>0.360$ ).

First, evaluations of overall RT revealed that the competitive group took longer and gathered more information $[\mathrm{M}=7.663, \mathrm{SE}=0.029$, $95 \%$ CI $(7605 ; 7.720)]$ in the dynamic task than the non-competitive group $[\mathrm{M}=7.496, \mathrm{SE}=0.032$, 95\% CI (7.434; 7.559], $p<.001)$, and thus needed more time and intensity of facial information for correct emotion categorization (Fig. 3A). Only descriptively, gathering of facial information to masked $\mathrm{FBO}$ was longer $[\mathrm{M}=7.608, \mathrm{SE}=0.031,95 \%$ CI $(7.548 ; 7.668)]$ than RTs to masker odor $[\mathrm{M}=7.551, \mathrm{SE}=0.031$, 95\% CI (7.491; 7.611), $p=.183$; Fig. 3B].

Based on our initial hypotheses, we checked the planned contrast on the joint effect of group and odor exposure (Fig. 3C). During masked FBO exposure, RTs of the competitive group $[\mathrm{M}=7.719, \mathrm{SE}=0.041$, $95 \%$ CI $(7.639 ; 7.800)]$ were longer than for the non-competitive group $[\mathrm{M}=7.497, \mathrm{SE}=0.045,95 \% \mathrm{CI}(7.409 ; 7.585), p<.001]$. During exposure to masker odor, RTs of the competitive group $[\mathrm{M}=7.606$, $\mathrm{SE}=0.042 ; 95 \% \mathrm{CI}(7.525 ; 7.688)]$ and RTs of the non-competitive group were not statistically different $[\mathrm{M}=7.495, \mathrm{SE}=0.045,95 \% \mathrm{CI}$ (7.407; 7.583), $p=.069$ ]. Without odor, sex or group effects, categorization RTs to all angry faces $(\mathrm{M}=7.720, \mathrm{SE}=0.031 ; 95 \% \mathrm{CI}[7.659$; 7.780]) were longer than categorization RTs to all happy faces $(\mathrm{M}=7.439, \mathrm{SE}=0.030 ; 95 \% \mathrm{CI}[7.380 ; 7.499])$.

Altogether, results show that competitive women process dynamic facial expressions more thoroughly (i.e. longer) than non-competitive women.

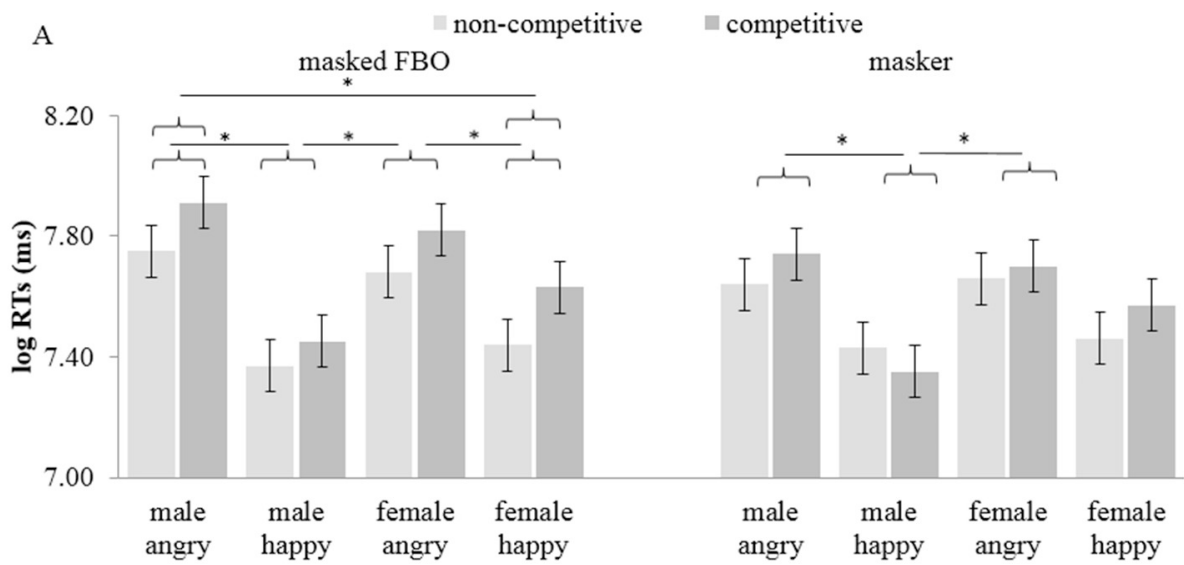

Fig. 4. Log-transformed RTs (M and SE) in the dynamic emotion categorization task. Upper panel (A) depicts all image categories (faces: male angry, male happy, female angry and female happy) by odor (left panel: masked FBO; right panel: masker) and direct comparison of competition groups. Lower panel (B) depicts all image categories grouped by competition group and direct comparison of odor conditions. * marks significance at $\mathrm{p}<.05$.

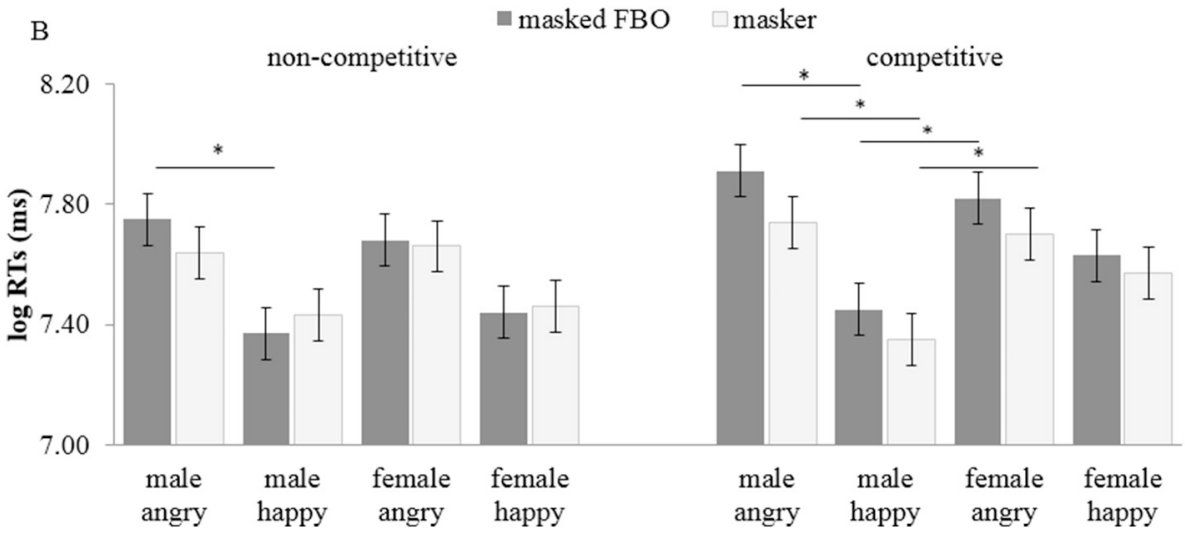


There is a similar tendency towards competitive women with female body odor exposure to process dynamic facial expressions more thoroughly than non-competitive women with female body odor exposure. Angry faces required longer categorization time than happy faces, irrespective of the sex of the image. In response to the above mentioned results, an explorative evaluation with specific focus on happy and angry emotions in female faces and then male happy and angry faces is reported in the supplementary material C. Graphs depicting RT with regards to group, odor, emotion, and sex of the face can be found in Fig. 4 .

\subsection{Irrespective of group and odor exposure, female happy faces were} associated with the strongest lapses of attention

With regards to the third hypothesis, specific effects on RT dispersion were analyzed. More lapses of attention (i.e., greater tau evident in the exponential tail of the ex-Gaussian distribution) were predicted under female body odor exposure. Further distribution analyses (i.e., mu and sigma) can be found in supplementary material C.

With respect to RT dispersion via Tau (negative skewness in the slopes), a main effect of image category $[\mathrm{F}(3,387)=8.905, p<.001]$ appeared (all other factors, including odor exposure and competition group, $p s>.161)$. Female happy faces $[\mathrm{M}=724.013$, $\mathrm{SE}=41.907$, $95 \%$ CI $(641.625 ; 806.406)]$, were associated with the largest tau compared to male angry faces $[\mathrm{M}=711.359, \mathrm{SE}=41.705,95 \% \mathrm{CI}$ (629.363; 793.355), $p<.001]$, to female angry faces $[M=696.608$, $\mathrm{SE}=41.502,95 \% \mathrm{CI}(615.011 ; 778.205), p<.001]$, and to male happy faces $[\mathrm{M}=461.776, \mathrm{SE}=41.907,95 \%$ CI (379.389; 544.169), $p<.001]$ respectively. While the largest difference appeared between female and male happy faces $(p<.001)$, no differences were seen between female and male angry faces $(p>$.999).

Without the influence of odor exposure or competition group, the greatest lapses of attention were seen in female happy faces. Lapses of attention did not occur in relation to female body odor exposure.

\subsection{When exposed to female body odor, competitive women showed stronger IHR activity}

The last hypothesis foresaw resistance to cardiac deceleration in the competitive vs. non-competitive group, particularly when exposed to the masked body odor of other women.
Positive values depict higher IHR activity and negative values describe IHR decelerations (more details of the LMM analysis to be found in supplementary material B). Results are presented in Table 2. Main effects of sampling time at $\mathrm{ms} 0$ to $6000[\mathrm{~F}(3,3577)=11.591$, $p<.001]$ and odor exposure (masker vs. masked FBO) [F $(1,3577)=8.766, p=.003]$ and an interaction of odor exposure $\mathrm{x}$ competition group $[\mathrm{F}(1,3577)=4.207, p=.040]$ appeared (all other $p_{s}>$.398). For the odor exposure at odor onset, no changes in IHR after the first bin were seen (ms 0 to 1999) $[\mathrm{M}=-0.140, \mathrm{SE}=0.179$, $95 \%$ CI $(-0.492 ; 0.211) p>$. 999]. Throughout the second and third sampling bins, significant IHR reductions were found (for ms 2000 to $3999[\mathrm{M}=-0.716, \mathrm{SE}=0.179,95 \%$ CI $(-1.068 ; 0.365) p=.029$; and for ms 4000 to $6000[\mathrm{M}=-1.335, \mathrm{SE}=0.179,95 \% \mathrm{CI}(-1.686$; $-0.984) p<.001]$ ).

For the competitive group, IHR to masked FBO was significantly higher compared to masker [M $\Delta=0.897, \mathrm{SE} \Delta=0.258, p=.001,95 \%$ CI $(0.391 ; 1.404)]$. For the non-competitive group, no IHR differences in association to masked FBO and masker were yielded $[\mathrm{M} \Delta=0.163$, $\mathrm{SE}=0.248, p=.512,95 \% \mathrm{CI}(-0.324 ; 0.649)]$. Looking at the separate bins for the competitive group, significantly higher IHR in association with masked $\mathrm{FBO}$ for all bins $\left[\mathrm{M} \Delta_{2000}=1.220, \mathrm{SE}=0.517\right.$, $p=.018 ; 95 \%$ CI $(0.207 ; 2.233)$ vs. $\mathrm{M} \Delta_{4000}=1.1720, \mathrm{SE}=0.517$, $p=.023,95 \%$ CI $(0.159 ; 2.184)$ vs. $\mathrm{M} \Delta_{6000}=1.1198, \mathrm{SE}=0.517$, $p=.020,95 \%$ CI $(0.185 ; 2.211)]$ were yielded (Fig. 5). For the noncompetitive group across separate bins, no significant differences in relation to both odor exposures were evident (all $p_{s}>.748$ ).

Across both competition groups, IHR in association with the masker odor depicted comparable IHR curves. Only in the competitive group, masked FBO was associated with a nominal increase in IHR in the first bin and a resistance to cardiac deceleration in the subsequent bins, suggesting a possible greater amount of activation and focused attention in the highly competitive state and exposure to female body odor compatible with defensive behavior.

\section{Discussion}

\subsection{General discussion}

The goal of this experiment was to determine whether women would categorize dynamic facial expressions differently based on a

Table 2

Baseline-controlled IHR (in ms, M and SE) with odor conditions (masked FBO and Masker) and competition groups averaged across bins of $2000 \mathrm{~ms}$ each. Significant comparisons are flagged via asterisks in Fig. 5.

\begin{tabular}{|c|c|c|c|c|}
\hline \multirow[t]{2}{*}{ Baseline controlled IHR (ms) } & \multicolumn{2}{|c|}{ Non-competitive group } & \multicolumn{2}{|c|}{ Competitive group } \\
\hline & Masked FBO & Masker & Masked FBO & Masker \\
\hline 2000 & $0.068(0.351)$ & $-0.092(0.351)$ & $0.342(0.365)$ & $-0.878(0.366)$ \\
\hline 4000 & $-0.694(0.351)$ & $-1.046(0.351)$ & $0.023(0.365)$ & $-1.148(0.366)$ \\
\hline 6000 & $-1.269(0.351)$ & $-1.408(0.351)$ & $-0.733(0.365)$ & $-1.931(0.366)$ \\
\hline
\end{tabular}
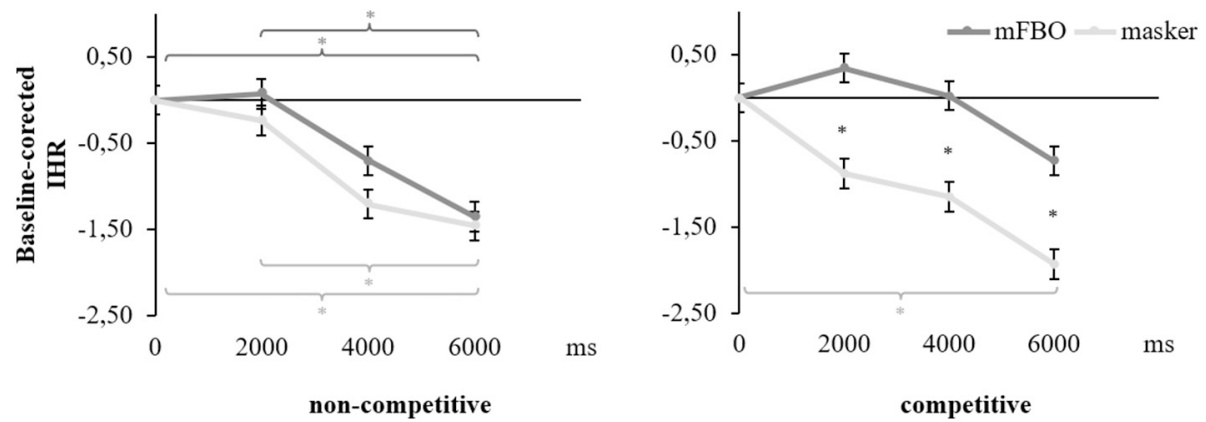

Fig. 5. Deceleration curves of the instantaneous heart rate analysis for odor conditions (masked FBO and masker) and separated by competition groups (noncompetitive and competitive). Temporal resolution in bins of $2000 \mathrm{~ms}$, depicting IHR after odor exposure (0 ms). Significant differences marked with *. 
competitive vs. non-competitive mindset, when exposed to body odors of stranger female donors. To elucidate the mechanisms behind women's subtle transmission of competitive and chemosensory cues, we have developed an experimental design to investigate female intrasexual competition and its modulations through the performance of a dynamic emotional face categorization task under the exposure of body odors of intrasexual competition. In line with our exploratory hypotheses, we reveal that:

First, a more competitive mindset (measured via competitiveness self-rating and response accuracy to the dynamic emotion categorization task) was observed in the competitive vs. the non-competitive group. This manipulation check indicates that experimental induction of competition in receivers was successful.

Second, competitive women displayed modulated emotion categorization RT. Descriptively, competitive women during exposure to female body odor processed dynamic facial expressions more thoroughly (i.e. longer) than non-competitive women. Additionally, we could associate all angry faces with longer categorization times.

Third, and in contrast to our assumptions, lapses of attention did not occur more frequently in relation to female body odor exposure. Instead, they were observed with female happy faces.

Fourth, women acting in a competitive framework combined with female body odor exposure displayed resistance to cardiac deceleration via IHR activity that can be translated into a greater amount of pressure and focused attention typical of defensive behavior.

Although exploratory, this research provides novel information on the subtle nature of female intrasexual communication through body odor and competition. Our key results support the notion that simultaneous processing of body odors can modify emotional face categorization and IHR activity in a competitive scenario. In our study, the key results hint towards body odor cues as a suitable method for investigating subtle modulations of female interpersonal communication (i.e., a competitive scenario). Although, the RT results do not fully support the assumption that female body odors modulate emotion categorization in a competitive situation, we see a separate significant effect of competition group (i.e., longer gathering of information) and a descriptive tendency towards extended emotion categorization when competitive women were exposed to female body odor. Nevertheless, the assumption that female body odors modulate emotion categorization in a competitive situation is supported by impaired cardiac deceleration in competitive women exposed to female body odor. Although one may argue that fast detection is the superior outcome in competition, we would like to point out that short RT are associated with higher decision insecurity in our set-up with a dynamic task. In contrast, longer RT equal more emotional expression and informational grounds for correct decision making. Therefore, we argue that a longer emotion processing is associated with more thorough attentional processing of relevant stimuli.

Two unexpected results deserve further attention. First, we observed longer processing latencies with angry faces vs. happy faces. This result can be translated into a higher granted focus on the facial expression, perhaps in virtue of its potential threatening nature. It is in line with previous research on static faces that show that happy faces were associated with shorter processing latencies (e.g., [33], [49] for a metaanalysis). Second, lapses of attention were associated with female happy faces. Although not specifically targeted in our research, frequent negatively skewed RT distributions might stand in association with impaired attentional disengagement from female happy faces. This result is in line with intrasexual competition research, finding that women explore positive visual features in other women's faces more thoroughly [22] and even prefer them in comparison to male faces [54]. All in all, results from static emotional faces are very common and not always comparable to results yielded from dynamic emotional faces (for a comparison, see [11]).

To reinforce the idea that the present results are not dependent on the between-subject nature of this experimental design (when it comes to the verbal induction of competition), the women included in the competitive and in the non-competitive groups show similar demographics, emotional and, trait competition states. Furthermore, olfactory ratings confirmed that the two applied odors showed similar intensity, pleasantness, familiarity, threat, and disgust ratings across groups.

\subsection{Chemosensory and psychophysiological effects in relation to competition in women}

Based on the present results, we can suggest that - besides the induction of a competitive framework, which capitalizes more on the cognitive appraisal of potential competition - the body odor of other women simulated competition via a sensory channel. This is not a new idea: scientists are uncovering more and more often that the olfactory system has evolved behavioral strategies to protect an odor receiver from threat in various forms: Receivers tend to engage in disgust reaction to avoid contagion when sniffing the body odor of unhealthy individuals [50] or remain in a freeze reaction to assess an imminent threat when sniffing the body odor of a dangerous and aggressive individual [47]. Providing more insight into the field of chemosensory research and as a more specific account on intrasexual effects, the present results indicate that competitive women exposed to the scent of another female show an autonomic nervous system reaction. Stemming from a chemosensory and emotional standpoint, this reaction could be translated into an increase of bodily vigilance associated with a cognitive freeze reaction in the emotion categorization and might be expressing the need to assess the degree of potential threat/competition in the vicinity. This assumption appears plausible as, in a broader chemosignaling context, women are known to be more receptive to subtle chemosensory signals in emotional contexts [56,59], perchance more strongly interfering with the dominant visual information. Vice versa, the female body odor is able to induce a chemosensory judgment effect towards a facilitated perception of feminine features in social and emotional tasks [46].

In our study, masking of the odors and using a dynamic facial categorization paradigm allowed us to test the effect of female body odors on competition in a more ecological context than previously tested. With respect to the masking of female body odor, this study provided an innovative method and stands out in comparison to other body odor studies. Common olfactory control conditions, within the research of female donors and receivers, have not been implemented by using an olfactory masker but by using a contrast of different body odor conditions, e.g., female body odor issuing from different menstrual cycle phases $([21,30,43])$. Although one may argue that incorporation of a fragrance might lead to a potential confound, this would, however and if anything, be included as a standard error common to both measurements. It is not to omit that body odor masking represents a more ecological approach, given real-life experiences where body odors are most commonly minimized by the use of other fragrances. Most practically, we have not found any effects of our masker odor. This setup does not claim to fully elucidate whether applications of other human body odors are able to produce further distinguishable effects (e.g., intersexual effects of male body odor), but it sheds light on the majorly under-investigated field of body odor contributions in purely female social interactions.

Female participants were particularly suitable for this specific study of psychophysiological processes of competition for several reasons: First, they were sufficiently sensitive to contextual odor cues in a nonhypothetical competitive context (supporting research ambitions presented by [76]). Second, they were seen to have stronger cardiovascular responses to an induced competitive motivation (in accordance with [73]). Third, under our most critical condition, a competitive framework coupled with female body odor, women displayed the strongest somatic responses: Comparable cardiac reactions were associated with the anticipatory stress of making a critical decision in the Iowa 
Gambling Task study [45]. The researchers found higher HR activity to be linked to the negative emotional valence of a behavior. In the present study, the nominally increased HR - strongest after imminent odor onset and stable in magnitude over the course of time - might be interpreted as a somatic marker of competition that validates our experimental manipulation. Importantly, competition-related responses were also seen boldly modified by high trait anxiety, similar to results reported in a physical performance competition [23], which points to the question of whether sensitivity to cognitive and chemosensory intrasexual competition might be further characterized by a receiver's anxiety level.

\section{Limitations}

Although the study was meticulously designed, some methodological aspects represent an opportunity for improvement in future studies. While the hormonal contraception intake was kept controlled for the receivers, future studies could specifically investigate possible effects in relation to the menstrual cycle phase of the receivers [18]. Human body odor communication, though, is not a prerogative of intersex interactions. Indeed, akin to men, women might also detect the odor cues to the menstrual cycle phase of another woman via body odor: Even in a small group of female raters, a tendency for the preference to the body odor of ovulating women [30] was seen and suggests that women can detect the reproductive status of other women via body odors. In contrast, the body odor of ovulating women was associated with higher testosterone levels in female raters, although without rating effects [40]. The physical presence of experimenters during testing can be considered as olfactory noise, although controlled across conditions.

Second, due to the study's explorative nature, our donation sample consisted of a rather small number of donors and our receivers were chosen upon strict inclusion criteria. With all receivers being exposed to the supra-donor stimuli, we minimized the effects of interpersonal differences in the presentation of body odors. Specifically, small groups of donors combined with long collection periods have the advantage of keeping the chemosensory signals rather stable over time by reducing inter-subject variability. We cannot fully exclude that unidentified idiosyncrasies related to some of the female donors or that the comparison of our receivers' sample with typical (student) populations might hinder generalizability of the presented data. Larger samples of donors are nevertheless warranted to confirm the present results and to further explore the influence of competitiveness communicated from female senders, e.g., by additionally separating the donors in a high and low competitiveness group. Third, the incorporation of the oakmoss fragrance as a possible confound (see above) has been carefully controlled by recruiting donors and receivers that were not expected to be familiar with each other and by applying the oakmoss masker on the clean pad (not on the body odor pad). In the data, odor rating results confirm that indeed no intensity, pleasantness and familiarity issues were retrieved.

Furthermore, some statistical limitations deserve heightened attention. Despite a reasonable length of the dynamic emotion categorization task (approx. $20 \mathrm{~min}$ ), similarly to other task durations [63], and the fact that we randomized item presentation, we cannot fully exclude the possibility that fatigue or improved performance through learning might have affected RT. Statistically, modeled time-on-task effects constitute an elegant way to rule out such concern but were not projected in the construction of our data acquisition at the time of experimental set up and we did not proceed to this analysis for fear of incurring in power issues.

One strategy to analyze RT data has been to approach them as exgaussian distributions. This method has the advantage of letting to emerge, via the analysis of the parameter tau (the exponential tail of the distribution) potential lapses of attention, as classically revealed when analyzing the performance of individuals with ADHD. In ADHD patients, the tau parameter was seen qualitatively different compared to healthy patients and was interpreted as an unreliable effort control mechanism during information processing explicitly expressed with attentional lapses [34]. Other models used to analyze RT distributions, such as drift diffusion models, provide a more holistic approach to cognitive information processing (e.g., including the motor responses, tracking of the accumulation of the information and the choice made; [42]). Considering that the present design was not optimized for the amount of information needed by drift diffusion models to converge (i.e., hundreds of trials per condition), we chose tau as the parameter of choice to reflect odor-dependent attentional changes, even if embedded in a less sophisticated model with regards to the statistical null results and claims to absence of effects, the use of Bayesian statistics can help further understand the strength of the findings highlighted, particularly since it provides a more robust method to prevent false positives findings. Especially with regards to RT-related hypotheses analyzed via LMMs, those methods might support the detection of possibly spurious results, which in the current analyses are significant but close to the .050 significance cut-off. We would like to encourage further research with expertise in Bayesian methods to fill this gap.

By exploring a fairly under-investigated field of female-female competitive behavior in combination with one of our most archaic senses, the present study sheds light on the emergence of subtle hints of rivalry and competition in social situations. Women's body odors implicitly suggesting the presence of another female - might, when presented in a competition-inducing context, be associated with modulated effects of emotional face detection and autonomic nervous processing. This being a study on intrasexual effects, it remains to elucidate whether dynamic emotion categorization is modified differently by the olfactory presence of a male odor source, and as an excellent opportunity for research in the role of social body odors in an intersexual competitive mating context.

\section{Declaration of interest}

none.

\section{Funding}

This work was supported by the Interdisciplinary Center for Clinical Research within the Faculty of Medicine at RWTH Aachen University, Germany (531214, N4-3) and the German Academic Exchange Service (DAAD).

\section{Disclosure statement}

The authors have nothing to disclosure.

\section{Appendix A. Supplementary data}

Supplementary data to this article can be found online at https:// doi.org/10.1016/j.physbeh.2019.112562.

\section{References}

[1] D. Adolph, S. Schlösser, M. Hawighorst, B.M. Pause, Chemosensory signals of competition increase the skin conductance response in humans, Physiol. Behav. 101 (5) (2010) 666-671.

[2] C. Allen, J. Havlíček, S.C. Roberts, Effect of fragrance use on discrimination of individual body odor, Front. Psychol. 6 (2015).

[3] R.M. Bagby, J.D. Parker, G.J. Taylor, The twenty-item Toronto Alexithymia Scale-I. Item selection and cross-validation of the factor structure, J. Psychosom. Res. 38 (1) (1994) 23-32.

[4] W.A. Babchuk, R.B. Hames, R.A. Thompson, Sex differences in the recognition of infant facial expressions of emotion: the primary caretaker hypothesis, Evol. Hum. Behav. 6 (2) (1985) 89-101.

[5] D.A. Balota, M.J. Yap, Moving beyond the mean in studies of mental chronometry, Curr. Dir. Psychol. Sci. 20 (2011) 160-166.

[6] N.J. Bean, C.J. Wysocki, Vomeronasal organ removal and female mouse aggression: 
the role of experience, Physiol. Behav. 45 (1989) 875-882.

[7] J.F. Benenson, The development of human female competition: allies and adversaries, Philos. Trans. R. Soc. B 368 (1631) (2013) 20130079.

[8] N.J. Briton, J.A. Hall, Beliefs about female and male nonverbal communication, Sex Roles 32 (1-2) (1995) 79-90.

[9] A.P. Buunk, M. Fisher, Individual differences in intrasexual competition, J. Evol. Psychol. 7 (1) (2009) 37-48.

[10] W.S. Cain, Odor identification by males and females: predictions vs performance, Chem. Senses 7 (1982) 129-142.

[11] M.G. Calvo, P. Avero, A. Fernández-Martín, G. Recio, Recognition thresholds for static and dynamic emotional faces, Emotion 16 (8) (2016) 1186.

[12] A. Campbell, Female competition: Causes, constraints, content, and contexts, J. Sex Res. 41 (1) (2004) 16-26.

[13] C. Cecchetto, A. Cavazzana, A.R. Gordon, V. Rebeschini, V. Parma, J.N. Lundström, Chemosensory Threat Signals: the Case of Strangers' Body Odor. In chemical senses (Vol. 41, no. 7, pp. E39-E40). Great Clarendon St, Oxford Ox2 6dp, Oxford Univ Press, England, 2016, September.

[14] C. Cecchetto, R.I. Rumiati, M. Aiello, Alexithymia and emotional reactions to odors, Sci. Rep. 7 (1) (2017) 14097.

[15] J.H. de Groot, G.R. Semin, M.A. Smeets, Chemical communication of fear: a case of male-female asymmetry, J. Exp. Psychol. Gen. 143 (4) (2014) 1515.

[16] B. Derntl, V. Schöpf, K. Kollndorfer, R. Lanzenberger, Menstrual cycle phase and duration of oral contraception intake affect olfactory perception, Chem. Senses 38 (2012).

[17] R.L. Doty, S. Applebaum, H. Zusho, R.G. Settle, Sex differences in odor identification ability: a cross-cultural analysis, Neuropsychologia 23 (1985) 667-672.

[18] R.L. Doty, E.L. Cameron, Sex differences and reproductive hormone influences on human odor perception, Physiol. Behav. 97 (2) (2009) 213-228.

[19] F. Faul, E. Erdfelder, A.-G. Lang, A. Buchner, G*power 3: a flexible statistical power analysis program for the social, behavioral, and biomedical sciences, Behav. Res. Methods 39 (2007) 175-191.

[20] M.L. Fisher, Female intrasexual competition decreases female facial attractiveness, Proc. R. Soc. Lond. B Biol. Sci. 271 (2004) S283-S285.

[21] K.A. Gildersleeve, M.G. Haselton, C.M. Larson, E.G. Pillsworth, Body odor attrac tiveness as a cue of impending ovulation in women: evidence from a study using hormone-confirmed ovulation, Horm. Behav. 61 (2) (2012) 157-166.

[22] J.A. Hall, Nonverbal Sex Differences: Communication Accuracy and Expressive Style, The Johns Hopkins University Press, Baltimore, MD, 1984.

[23] P. Hassmén, N. Koivula, Cardiac deceleration in elite golfers as modified by noise and anxiety during putting, Percept. Mot. Skills 92 (2001) 947-957.

[24] J. Havlíček, R. Dvořáková, L. Bartoš, J. Flegr, Non-advertized does not mean concealed: body odour changes across the human menstrual cycle, Ethology 112 (1) (2006) 81-90.

[25] A.S. Hervey, J.N. Epstein, J.F. Curry, S. Tonev, L. Eugene Arnold, C. Keith Conners, et al., L. Hechtman, Reaction time distribution analysis of Neuropsychologica performance in an ADHD sample, Child Neuropsychol. 12 (2) (2006) 125-140.

[26] H. Hoffmann, H. Kessler, T. Eppel, S. Rukavina, H.C. Traue, Expression intensity, gender and facial emotion recognition: women recognize only subtle facial emotions better than men, Acta Psychol. 135 (3) (2010) 278-283.

[27] T. Hummel, B. Sekinger, S. Wolf, E. Pauli, G. Kobal, Sniffin'sticks': olfactory performance assessed by the combined testing of odor identification, odor discrimination and olfactory threshold, Chem. Senses 22 (1) (1997) 39-52.

[28] G. Jones, A. Swain, Intensity and direction as dimensions of competitive state an xiety and relationships with competitiveness, Percept. Mot. Skills 74 (2) (1992) 467-472.

[29] G. Kobal, T. Hummel, B. Sekinger, S. Barz, S. Roscher, S. Wolf, Sniffin'sticks: screening of olfactory performance, Rhinology 34 (4) (1996) 222-226.

[30] S. Kuukasjärvi, C.P. Eriksson, E. Koskela, T. Mappes, K. Nissinen, M.J. Rantala, Attractiveness of women's body odors over the menstrual cycle: the role of oral contraceptives and receiver sex, Behav. Ecol. 15 (4) (2004) 579-584.

[31] P. Lenochová, S.C. Roberts, J. Havlíček, Methods of human body odor sampling: the effect of freezing, Chem. Senses (2) (2009) 34

[32] P. Lenochová, P. Vohnoutova, S.C. Roberts, E. Oberzaucher, K. Grammer, J. Havlíček, Psychology of fragrance use: perception of individual odor and perfume blends reveals a mechanism for idiosyncratic effects on fragrance choice, PLoS ONE 7 (3) (2012) e33810.

[33] J.M. Leppänen, J.K. Hietanen, Positive facial expressions are recognized faster than negative facial expressions, but why? Psychol. Res. 69 (1-2) (2004) 22-29.

[34] C. Leth-Steensen, Z.K. Elbaz, V.I. Douglas, Mean response times, variability, and skew in the responding of ADHD children: a response time distributional approach, Acta Psychol. 104 (2) (2000) 167-190.

[35] D. Lundqvist, J. Litton, The Averaged Karolinska Directed Emotional Faces-AKDEF, CD ROM from Department of Clinical Neuroscience, Psychology Section, Karolinska Institutet, (1998), (1998).

[36] J.N. Lundström, J.A. Boyle, R.J. Zatorre, M. Jones-Gotman, Functional neuronal processing of body odors differs from that of similar common odors, Cereb. Cortex 18 (6) (2008) 1466-1474.

[37] J.N. Lundström, J.A. Boyle, R.J. Zatorre, M. Jones-Gotman, The neuronal substrates of human olfactory based kin recognition, Hum. Brain Mapp. 30 (8) (2009) $2571-2580$.

[38] J.N. Lundström, M. Jones-Gotman, Romantic love modulates women's identification of men's body odors, Horm. Behav. 55 (2) (2009) 280-284.

[39] J.N. Lundström, A.R. Gordon, E.C. Alden, S. Boesveldt, J. Albrecht, Methods for building an inexpensive computer-controlled olfactometer for temporally-precise experiments, Int. J. Psychophysiol. 78 (2) (2010) 179-189.

[40] J.K. Maner, J.K. McNulty, Attunement to the fertility status of same-sex rivals: women's testosterone responses to olfactory ovulation cues, Evol. Hum. Behav. 34 (2013) 412-418.

[41] Y. Martins, G. Preti, C.R. Crabtree, T. Runyan, A.A. Vainius, C.J. Wysocki, Preference for human body odors is influenced by gender and sexual orientation, Psychol. Sci. 16 (9) (2005) 694-701.

[42] D. Matzke, E.J. Wagenmakers, Psychological interpretation of the ex-Gaussian and shifted Wald parameters: a diffusion model analysis, Psychon. Bull. Rev. 16 (5) (2009) 798-817.

[43] S.L. Miller, J.K. Maner, Scent of a woman men's testosterone responses to olfactory ovulation cues, Psychol. Sci. 21 (2) (2010) 276-283.

[44] S. Mitro, A.R. Gordon, M.J. Olsson, J.N. Lundström, The smell of age: perception and discrimination of body odors of different ages, PLoS ONE 7 (5) (2012) e38110, https://doi.org/10.1371/journal.pone.0038110.

[45] A.C. Miu, R.M. Heilman, D. Houser, Anxiety impairs decision-making: psychophysiological evidence from an Iowa gambling task, Biol. Psychol. 77 (3) (2008) 353-358.

[46] S. Mutic, E.M. Moellers, M. Wiesmann, J. Freiherr, Chemosensory communication of gender information: masculinity bias in body odor perception and femininity bias introduced by chemosignals during social perception, Front. Psychol. 6 (2015) 1980.

[47] S. Mutic, V. Parma, Y.F. Brünner, J. Freiherr, You smell dangerous: communicating fight responses through human chemosignals of aggression, Chem. Senses 41 (41) (2016) 35-43.

[48] S. Mutic, Y.F. Brünner, R. Rodriguez-Raecke, M. Wiesmann, J. Freiherr, Chemosensory danger detection in the human brain: body odor communicating aggression modulates limbic system activation, Neuropsychologia 99 (2017) $187-198$.

[49] L. Nummenmaa, M.G. Calvo, Dissociation between recognition and detection advantage for facial expressions: a meta-analysis, Emotion 15 (2) (2015) 234-256.

[50] M.J. Olsson, J.N. Lundström, B.A. Kimball, A.R. Gordon, B. Karshikoff, N. Hosseini, et al., J. Axelsson, The scent of disease: human body odor contains an early chemosensory cue of sickness, Psychol. Sci. 25 (3) (2014) 817-823.

[51] D. Palomba, A. Angrilli, A. Mini, Visual evoked potentials, heart rate responses and memory to emotional pictorial stimuli, Int. J. Psychophysiol. 27 (1) (1997) 55-67.

[52] D. Palomba, M. Sarlo, A. Angrilli, A. Mini, L. Stegagno, Cardiac responses associated with affective processing of unpleasant film stimuli, Int. J. Psychophysiol. 36 (1) (2000) 45-57.

[53] J.D. Parker, G.J. Taylor, M. Bagby, Alexithymia and the recognition of facial expressions of emotion, Psychother. Psychosom. 59 (3-4) (1993) 197-202.

[54] V. Parma, R. Tirindelli, A. Bisazza, S. Massaccesi, U. Castiello, Subliminally perceived odours modulate female intrasexual competition: an eye movement study, PLOS ONE 7 (2) (2012).

[55] V. Parma, A.R. Gordon, C. Cecchetto, A. Cavazzana, J.N. Lundström, M.J. Olsson, Processing of human body odors, Springer Handbook of Odor, Springer, Cham, 2017, pp. 127-128.

[56] B.M. Pause, A. Ohrt, A. Prehn, R. Ferstl, Positive emotional priming of facial affect perception in females is diminished by chemosensory anxiety signals, Chem. Senses 29 (9) (2004) 797-805.

[57] A.D. Pazda, P. Prokop, A.J. Elliot, Red and romantic rivalry viewing another woman in red increases perceptions of sexual receptivity, derogation, and intentions to mate-guard, Personal. Soc. Psychol. Bull. 40 (10) (2014) 1260-1269.

[58] D.A. Puts, J.L. Barndt, L.L. Welling, K. Dawood, R.P. Burriss, Intrasexual competition among women: vocal femininity affects perceptions of attractiveness and flirtatiousness, Personal. Individ. Differ. 50 (1) (2011) 111-115.

[59] A.R. Radulescu, L.R. Mujica-Parodi, Human gender differences in the perception of conspecific alarm chemosensory cues, PLoS ONE 8 (7) (2013).

[60] J. Rehnman, A. Herlitz, Higher face recognition ability in girls: magnified by ownsex and own-ethnicity bias, Memory 14 (3) (2006) 289-296.

[61] S.C. Roberts, J. Havlíček, J. Flegr, M. Hruskova, A.C. Little, B. Jones, C. et al. \& Petrie, M. Female facial attractiveness increases during the fertile phase of the menstrual cycle, Proc. R. Soc. Lond. B 5 (2004) S270-S272.

[62] M. Rocha, V. Parma, J. Lundström, S.C. Soares, Anxiety body odor induces a stress response in the recipient: evidence from recognition of dynamic facial expressions and heart rate variability, Chem. Senses 41 (2016).

[63] M. Rocha, V. Parma, J.N. Lundström, S.C. Soares, Anxiety body Odors as context for dynamic faces: categorization and psychophysiological biases, Perception 47 (10-11) (2018) 1054-1069.

[64] D. Singh, P.M. Bronstad, Female body odour is a potential cue to ovulation, Proc. R. Soc. Lond. B Biol. Sci. 268 (1469) (2001) 797-801.

[65] P.B. Singh, A. Young, S. Lind, M.C. Leegaard, A. Capuozzo, V. Parma, Smelling anxiety chemosignals impairs clinical performance of dental students, Chem. Senses 43 (6) (2018) 411-417.

[66] P. Sorokowski, M. Karwowski, M. Misiak, M.K. Marczak, M. Dziekan, T. Hummel, A. Sorokowska, Sex differences in human olfaction: a meta-analysis, Front. Psychol. 10 (2019) 242.

[67] C.D. Spielberger, Manual for the State-Trait Anger Expression Inventory-2, Psychological Assessment Resources, Odessa, FL, 1999.

[68] S.E. Taylor, L.C. Klein, B.P. Lewis, T.L. Gruenewald, R.A. Gurung, J.A. Updegraff, Biobehavioral responses to stress in females: tend-and-befriend, not fight-or-flight, Psychol. Rev. 107 (3) (2000) 411-429. 
[69] R. Tirindelli, M. Dibattista, S. Pifferi, A. Menini, From pheromones to behavior, Psychol. Rev. 89 (3) (2009) 921-956.

[70] T. Vaillancourt, Do human females use indirect aggression as an intrasexual competition strategy? Philos. Trans. R. Soc. Lond. B 368 (1631) (2013) 20130080.

[71] T. Vaillancourt, A. Sharma, Intolerance of sexy peers: intrasexual competition among women, Aggress. Behav. 37 (6) (2011) 569-577.

[72] N.T. Van Dam, D.F. Gros, M. Earleywine, M.M. Antony, Establishing a trait anxiety threshold that signals likelihood of anxiety disorders, Anxiety Stress Coping 26 (1) (2013) 70-86.
[73] L.F. Van Egeren, Cardiovascular changes during social competition in a mixedmotive game, J. Pers. Soc. Psychol. 37 (6) (1979) 858.

[74] R. Whelan, Effective analysis of reaction time data, Psychol. Rec. 58 (3) (2010) 9

[75] T.S. Wingenbach, C. Ashwin, M. Brosnan, Sex differences in facial emotion recognition across varying expression intensity levels from videos, PLoS ONE 13 (1) (2018) e0190634.

[76] S.L. Woodward, M.E. Thompson, S.W. Gangestad, Women exposed to the scents of fertile-phase and luteal-phase women: evaluative, competitive, and endocrine responses, Adapt. Hum. Behav. Physiol. 1 (4) (2015) 434-448. 